\title{
Interference-Aware Opportunistic Relay Selection
}

\author{
Tauseef Jamal, Paulo Mendes \\ SITI, University Lusofona \\ \{tauseef.jamal,paulo.mendes\}@ulusofona.pt
}

\author{
André Zúquete \\ University of Aveiro \\ andre.zuquete@ua.pt
}

\section{INTRODUCTION}

Recently, cooperative relaying techniques have been investigated to increase the performance of wireless systems by using diversity created by different single antenna devices. With the introduction of cooperative relaying, relay selection requires special attention, since it has a strong impact on transmission performance. Moreover, cooperation procedures may be very unstable when relay selection relies on current channel conditions and is agnostic of interference induced by simultaneous transmissions. As a result, the performance of cooperative relaying in realistic scenarios may be very different from interference-free scenarios that have been the focus of most of the existing proposals so far.

Aiming to support cooperation in realistic scenarios, we analyze the impact of an opportunistic relay selection mechanism that is aware of interference and agnostic of channel status. With the proposed scheme, potential relays opportunistically configure their contention window based on an on-the-fly analysis of direct and indirect interference, instead of node degree or distance towards the destination [1]. The proposed opportunistic relay selection mechanism is part of a framework, called RelaySpot [2], which implements a cooperative wireless solution for large mobile networks, based upon the combination of opportunistic relay selection, cooperative scheduling and chain relaying.

\section{INTERFERENCE-AWARE OPPORTUNIS- TIC RELAY SELECTION}

Node degree gives an indication about the probability of having successful relay transmissions: having information about the number of neighbors allows the minimization of the collision risk, as well as blockage of resources. However, it is possible that nodes with

\footnotetext{
Permission to make digital or hard copies of all or part of this work for personal or classroom use is granted without fee provided that copies are not made or distributed for profit or commercial advantage and that copies bear this notice and the full citation on the first page. To copy otherwise, to republish, to post on servers or to redistribute to lists, requires prior specific permission and/or a fee.

ACM CoNEXT Student Workshop, December 6, 2011, Tokyo, Japan. Copyright 2011 ACM 978-1-4503-1042-0/11/0012 ...\$10.00.
}

low degree are overloaded due to: i) local processing of transmissions to and from neighbor nodes (direct interference); ii) influence of concurrent transmissions among neighbor nodes (indirect interference). Hence, our approach does not rely solely upon node degree, but takes also into account the traffic load of transmissions to and from neighboring nodes, to compute the overall interference level that each node is subjected to.

Equation 1 estimates the interference level that a potential relay is subjected to as a function of node degree and load. Let $\mathrm{N}$ be the number of neighbors of a potential relay, $T_{d}$ and $T_{i}$ the propagation time of direct and indirect transmissions associated to the potential relay, respectively, and $N_{i}$ and $N_{d}$ the number of nodes involved in such indirect and direct transmissions. Adding to this, $T_{p}$ is the time required for a potential relay to process the result of a direct transmission. The interference factor (I) affecting a potential relay has a minimum value of zero corresponding to no direct or indirect transmissions.

$$
I=\sum_{j=1}^{N_{d}}\left(T_{d j}+T_{p j}\right)+\sum_{k=1}^{N_{i}} T_{i k}, \quad I \in[0, \infty[
$$

The goal is to select as relay a node that has low interference factor, which means few neighbors (ensuring low blockage probability), and fast indirect and direct transmissions (ensuring low delays for packet relaying).

By using the interference level of a node, the probability of selecting such node as a relay is given by Equation 2 .

$$
\left.\left.S=\frac{1}{1+I}, \quad S \in\right] 0,1\right]
$$

After overhearing data packets towards a destination, a potential relay uses the estimated selection factor $(\mathrm{S})$ to compute the size of its contention window (CW), between a predefined minimum and maximum values of $C W_{\min }$ and $C W_{\max }$, as given by Equation 3 .

$$
C W=C W_{\min }+(1-S)\left(C W_{\max }-C W_{\min }\right)
$$

\section{EVALUATION AND DISCUSSION}




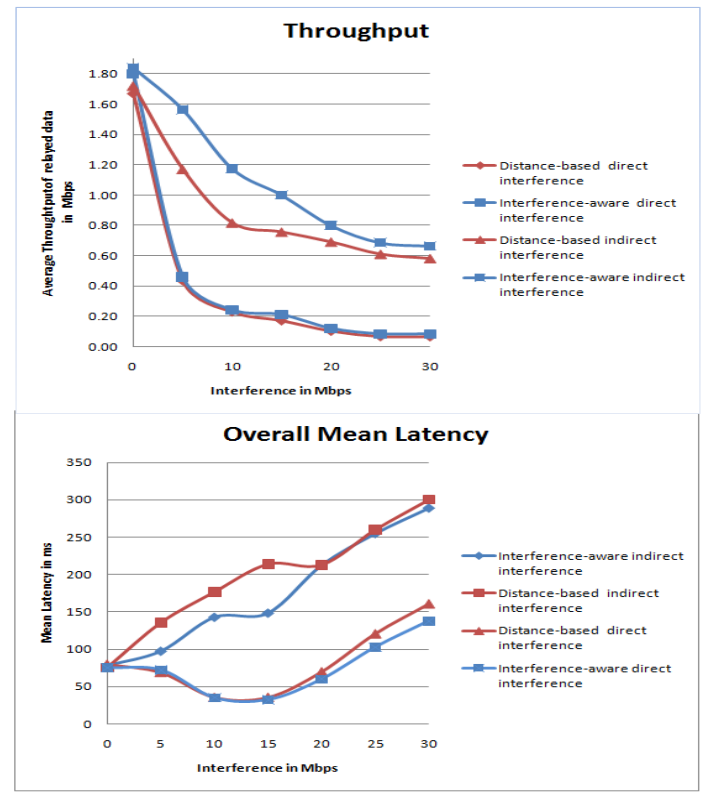

Figure 1: Throughput and Latency Analysis

In this section we evaluate the performance of the proposed interference-aware opportunistic relay selection mechanism in the presence of different levels of direct and indirect interference. Several simulations are run based on the MiXim framework of the OMNET++ 4.1 simulator. Each simulation has a duration of 300 seconds and is run ten times to provide a $95 \%$ confidence interval for the results.

Simulations consider a scenario where all nodes are static and have similar stochastic history of transmissions among them. The source and destination are at a distance of more than 150 meters from each other with a poor direct link, with an average of 1 Mbps. Network Allocation Vector (NAV) is used to compute $T_{d}$ and $T_{i}$, while $T_{p}$ is the average delay between packets extracted from MAC queue until it sense the channel.

Since prior-art is mostly based on node degree and distance towards a destination, we started by analyzing the performance of these approaches. Results show that distance-based approaches have better performance (e.g. throughput is 20 percentage points better in average), since in an interference-free scenario the best performance can be achieved by selecting as relay a node closer to the destination, even is such node has not the smallest neighbor set. Hence, we use the destinationbased approach as a benchmark.

Figure 1 shows that the proposed RelaySpot interferenceaware approach has better performance than distancebased approaches, with different traffic load (direct and indirect interference).

In a scenario with direct interference, the throughput gain of our approach is not significant. The gain of the interference-aware approach is more visible in the case of latency, since the interference-aware approach selects a relay from a set of nodes that present higher availability for retransmission (lower number of local generated traffic), even if placed further away from the destination, leading to lower latency. This gain is clearer with high traffic load, since distance-based approach keep selecting overloaded nodes near the destination.

In a scenario with indirect interference, the throughput gain of our interference-aware approach is significant (e.g. $33 \%$ with a load of 10 concurrent flows). With an increase of traffic load this performance gain diminishes, but is always higher than the distance-based approach. The advantage of our interference-aware approach is also visible in terms of latency, since selecting a relay with low interference (lower number of concurrent neighbor flows) leads to higher transmission opportunities. The gain in latency decreases with a load of 20 concurrent flows, mainly due to increasing number of concurrent flows placed faraway from the destination, which benefits distance-based approaches. Nevertheless, results show that even with a random placement of concurrent flows, the interference-aware solution keeps a lower latency with high traffic loads.

\section{CONCLUSIONS AND FUTURE WORK}

Most of the prior-art targets interference-free scenarios, which are not a realistic situation. Moreover such approaches are based on channel status estimations, which decreases their reliability. We propose an opportunistic relay selection mechanism that is aware of interference and agnostic of channel status. With the proposed scheme, potential relays opportunistically configure their contention window based on an analysis of direct and indirect interference. Simulation results show that relay selection based on the proposed interferenceaware scheme can provide performance improvement in relation to proposals based on node degree and distance. As a future work we will complement the interferenceaware approach by taking into account the percentage of successful retransmission of potential relays as well as their mobility pattern aiming to evaluate the overall RelaySpot proposal [2].

\section{ACKNOWLEDGMENT}

Thanks are due to ULOOP project (257418) and FCT for $\mathrm{PhD}$ grant (SFRH/BD/60436/2009).

\section{REFERENCES}

[1] N. Marchenko, E. Yanmaz, H. Adam, and C. Bettstetter, "Selecting a spatially efficient cooperative relay," in Proc. of IEEE GLOBECOM, (Honolulu, USA), Dec 2009.

[2] T. Jamal, P. Mendes, and A. Zuquete, "Relayspot: A framework for opportunistic cooperative relaying," in Proc. of IARIA ACCESS, (Luxembourg), June 2011. 